\title{
Terapia por fotobiomodulação: mecanismo de ação e importância clínica no tratamento da osteoartrite de joelho
}

\author{
Photobiomodulation therapy: mechanism of action and clinical importance of treating knee \\ osteoarthritis \\ Terapia de fotobiomodulación: mecanismo de acción e importancia clínica del tratamiento de la \\ osteoartritis de rodilla
}

Recebido: 18/01/2021 | Revisado: 23/01/2021 | Aceito: 25/01/2021 | Publicado: 31/01/2021

\author{
Yuri Victor Barbosa Silva \\ ORCID: https://orcid.org/0000-0003-3297-0119 \\ Universidade Brasil, Brasil \\ E-mail: yurifisiocenter@hotmail.com \\ Thiago Correia da Silva Rezzo \\ ORCID: https://orcid.org/0000-0001-5568-7688 \\ Universidade Brasil, Brasil \\ E-mail: thiagorezzo@gmail.com \\ Carla Roberta Tim \\ ORCID: https://orcid.org/ 0000-0002-4745-9375 \\ Universidade Brasil, Brasil \\ E-mail: carla.tim@universidadebrasil.edu.br \\ Patrícia Gabrielli Vassão \\ ORCID: https://orcid.org/0000-0001-8728-1842 \\ Universidade Federal de São Paulo, Brasil \\ E-mail: patriciavassao@gmail.com \\ Thalita Páez \\ ORCID: https://orcid.org/0000-0003-4314-0380 \\ Universidade Brasil, Brasil \\ E-mail: thalitatp@ hotmail.com \\ Lívia Assis Garcia \\ ORCID: https://orcid.org/0000-0002-8343-3375 \\ Universidade Brasil, Brasil \\ E-mail: livia.assis@universidadebrasil.edu.br
}

\begin{abstract}
Resumo
A osteoartrite do joelho (OAJ) é uma das doenças articulares degenerativa progressiva mais comuns em todo o mundo e também uma das principais causas de incapacidade e danos à qualidade de vida. A terapia de fotobiomodulação (PBMT, do inglês Photobiomodulation Therapy) através de laser de baixa intensidade (LLLT, do inglês Low Level Laser Therapy) tem mostrado ser um tratamento promissora e com bons resultados para diversas doenças crônicas, incluindo as alterações que acometem o sistema articular. Dentro deste contexto, o objetivo deste estudo é proporcionar, por meio de uma revisão narrativa, uma compreensão das evidências atuais disponíveis sobre o mecanismo de ação e importância clínica da PBMT a laser no tratamento da OAJ. As buscas foram realizadas nas bases de dados bibliográficas do PubMed/MEDLINE, Biblioteca virtual da saúde (BVS), Web of Science e SciELO. Com base nos estudos revisados por pares, os dados laboratoriais em modelos animais experimentais e estudos clínicos mostram que a PBM é capaz de reduzir a morte de condrócitos, estimular a síntese de componentes da matriz extracelular (MEC), atenuar a liberação de mediadores inflamatórios e proteolíticos presente em doenças articulares crônicas, prevenindo assim a degradação do tecido articular e periarticulares. Consequentemente, clinicamente, pode ser observado melhora da dor, funcionalidade e qualidade de vida do indivíduo acometido com OAJ e tratados com a PBMT a laser. Assim, podemos concluir que a PBMT a laser pode constituir um excelente coadjuvante no tratamento da OAJ, uma vez que é um método não invasivo e também apresenta custos relativamente baixos.
\end{abstract}

Palavras-chave: Osteoartrite; Reabilitação; Laser; Joelho.

\begin{abstract}
Osteoarthritis of the knee (OAJ) is one of the most common progressive degenerative joint diseases worldwide and also a major cause of disability and damage to quality of life. Photobiomodulation therapy (PBMT, from English Photobiomodulation Therapy) using low-level laser (LLLT, from English Low Level Laser Therapy) has shown to be a promising treatment with good results for several chronic diseases, including changes that affect the system articulate. Within this context, the objective of this study is to provide, through a narrative review, an understanding
\end{abstract}


of the current evidence available on the mechanism of action and clinical importance of laser PBMT in the treatment of OAJ. The searches were performed in the bibliographic databases of PubMed / MEDLINE, Virtual Health Library (VHL), Web of Science and SciELO. Based on peer-reviewed studies, laboratory data in experimental animal models and clinical studies show that PBM is able to reduce chondrocyte death, stimulate the synthesis of extracellular matrix components (ECM), mitigate the release of inflammatory mediators and proteolytics present in chronic joint diseases, thus preventing the degradation of articular and periarticular tissue. Consequently, clinically, improvement in pain, functionality and quality of life of the individual affected with OAJ and treated with laser PBMT can be observed. Thus, we can conclude that laser PBMT can be an excellent adjunct in the treatment of OAJ, since it is a non-invasive method and also has relatively low costs.

Keywords: Osteoarthritis; Rehabilitation; Laser; Knee.

\section{Resumen}

La osteoartritis de rodilla $(\mathrm{OAJ})$ es una de las enfermedades articulares degenerativas progresivas más comunes en todo el mundo y también una de las principales causas de discapacidad y daño a la calidad de vida. La terapia de fotobiomodulación (PBMT, de English Photobiomodulation Therapy) con láser de bajo nivel (LLLT, de English Low Level Laser Therapy) ha demostrado ser un tratamiento prometedor con buenos resultados para varias enfermedades crónicas, incluidos los cambios que afectan el sistema articular. En este contexto, el objetivo de este estudio es proporcionar, a través de una revisión narrativa, una comprensión de la evidencia actual disponible sobre el mecanismo de acción y la importancia clínica del láser PBMT en el tratamiento de la OAJ. Las búsquedas se realizaron en las bases de datos bibliográficas de PubMed / MEDLINE, Virtual Health Library (VHL), Web of Science y SciELO. Según estudios revisados por pares, los datos de laboratorio en modelos animales experimentales y estudios clínicos muestran que el PBM es capaz de reducir la muerte de condrocitos, estimular la síntesis de componentes de la matriz extracelular (ECM), mitigar la liberación de mediadores inflamatorios y proteolíticos presentes en enfermedades articulares crónicas. Evitando así la degradación del tejido articular y periarticular. En consecuencia, clínicamente se puede observar una mejoría del dolor, la funcionalidad y la calidad de vida del individuo afectado con OAJ y tratado con láser PBMT. Así, podemos concluir que el láser PBMT puede ser un excelente coadyuvante en el tratamiento de la OAJ, ya que es un método no invasivo y además tiene costos relativamente bajos.

Palabras clave: Artrosis; Rehabilitación; Láser; Rodilla.

\section{Introdução}

A osteoartrite de joelho $(\mathrm{OAJ})$ é uma doença articular crônica degenerativa complexa com múltiplos fatores de risco e patogênese complexa (Hussain et al., 2016). Caracteriza-se por um processo inflamatório crônico que provoca degeneração da cartilagem articular e outros tecidos periarticulares, como a cápsula articular, meniscos, sinóvia, músculos periféricos e ligamentos (Lespasio et al., 2019).

A perda progressiva de cartilagem articular, observada na OAJ pode causar dor, edema, fraqueza muscular, associada à instabilidade articular, perda de amplitude de movimento e redução da propriocepção, responsáveis por grande parte das incapacidades funcionais do indivíduo acometido (Hafez, et al., 2013; Hinman et al., 2020). Além disso, dados do Osteoarthritis Initiative Study indicaram que a OAJ pode ter um impacto negativo na saúde mental dos indivíduos acometidos (Vina \& Kwoh, 2018).

De acordo com as estatísticas atuais, cerca de $10 \%$ a $25 \%$ das pessoas com mais de 60 anos possuem OAJ (Hinman et al., 2020) e está associada a elevados custos socioeconômicos, o qual envolve gastos diretos com internações e medicações, além de gastos indiretos relacionados ao aumento da morbidade e de sintomas ligados a quadros depressivos e de ansiedade entre os acometidos (French et al., 2009). Mais seriamente, com a mudança demográfica para uma população mais envelhecida, o número de pessoas com OAJ e despesas financeiras também aumentam proporcionalmente (Turkiewicz et al., 2014).

A alta prevalência da OAJ e seu impacto socioeconômico e na qualidade de vida mostram a importância de mais pesquisas cujo os objetivos estão voltados para o desenvolvimento e investigação de estratégias de prevenção/tratamento para desta condição clínica (Benner et al., 2019). Sendo assim, vários recursos terapêuticos têm sido estudados na tentativa de 
prevenir e/ou diminuir o dano articular, bem como, amenizar os impactos inerentes a OAJ, tais como tais como a acupuntura (LU et al., 2010), termoterapia e a estimulação elétrica transcutânea (TENS) (Zhang et al., 2008).

Atualmente, vêm se destacando a terapia por fotobiomodulação (PBMT, do inglês Photobiomodulation Therapy) a laser de baixa intensidade (LLLT- Low Level Laser Therapy) no tratamento de pacientes com OAJ (Ferreira, Duarte \& Gonçalves, 2018).

Dentro deste contexto, o objetivo deste estudo é proporcionar uma compreensão das evidências atuais disponíveis a respeito dos mecanismos de ação e implicações clínicas da PBMT a laser no tratamento da OAJ.

\section{Referencial Teórico}

\section{Osteoartrite de joelho}

A OAJ é a forma mais comum de doença articular crônica, definida pela degeneração progressiva da matriz extracelular (MEC) do tecido cartilaginoso, remodelamento do osso subcondral e inflamação de todos os tecidos periarticulares (Hussain et al., 2016; Lespasio et al., 2019).

A etiologia da doença é multifatorial e os fatores de risco comuns incluem fatores físicos (idade, sexo feminino, obesidade, história familiar) e fatores mecânicos prejudiciais locais (trauma, desalinhamento articular e uso ocupacional e/ou recreativo) (Hussain et al., 2011).

Tem sido demonstrada que a OAJ ocorre devido a um desequilíbrio entre atividades anabólicas e catabólicas, com predomínio da degradação dos componentes da MEC (Shi et al., 2006). Após estímulos lesivos no tecido cartilaginoso, os condrócitos e células inflamatórias iniciam a sintetize de citocinas pró-inflamatórias e oxidantes, responsáveis por iniciar e perpetuar o processo degenerativo articular (Pelletier et al., 2000). Neste processo, as principais citocinas pró-inflamatórias envolvidas são: o fator de necrose tumoral (TNF- $\alpha$ ), a interleucina 1 beta (IL-1 $\beta$ ) e a interleucina (IL-6) (KAPOOR et al., 2011). O aumento da concentração dessas citocinas no tecido cartilaginoso estimula a produção de uma variedade de enzimas proteolíticas, como as metaloproteinases de matriz (MMPs), com destaque para a MMP-13, responsáveis por degradar colágeno e proteoglicanas. Em consequência, há uma redução de colágeno do tipo II e IX e o aumento dos colágenos do tipo I e III, modificando a qualidade e a função biomecânica da matriz cartilaginosa (Goldring \& Goldring 2006; Goldring \& Goldring 2004).

Com a progressão da doença, ou seja, na persistência do estímulo lesivo no tecido cartilaginoso, é possível observar alterações morfológicas características de degeneração dos constituintes articulares, identificadas por desorganização e consequente morte de condrócitos, fibrilações e microfissuras da cartilagem, assim como o remodelamento do osso subcondral e presença de osteófitos marginais (Goldring \& Goldring 2006; Renner et al., 2006).

Clinicamente, os sintomas da OAJ incluem dor, edema, rigidez, limitação da amplitude de movimento, diminuição na força muscular e progressiva perda funcional (Jones et al., 2012). Além disso, é possível ser observado um desalinhamento do ângulo do joelho (valgo e varo estático), hipomobilidade, deformidade e disfunção articular em alguns casos (Afsar et al., 2017). Contudo, o principal sintoma da OA é a presença de dor (Perrot, 2015).

Apesar dos resultados promissores e avanços em experimentos que utilizam técnicas de engenharia de tecidos para reparo da cartilagem, sua tradução para a prática clínica permanece um desafio (Xiang et al., 2020) Dentre estes, vêm se destacando mais recentemente a PBMT por laser de baixa intensidade, uma abordagem terapêutica não invasiva e eficiente para o tratamento clínico de diversas lesões musculoesqueléticas (Bjordal et al., 2010; Hegedus et al., 2009).

Sabe-se que esta terapia de luz monocromática, interage com fotorreceptores celulares (cromóforos) específicos e estimulam as propriedades bioquímicas das células, dentre as quais, podemos destacar o aumento da respiração celular e 
oxigenação tecidual, aumento da síntese de DNA, RNA e proteínas reguladoras do ciclo celular, que podem promover a proliferação celular (Hamblin et al., 2013; Trevisan et al., 2020).

Dentre os principais efeitos descritos na literatura referente à PBMT, pode-se destacar propriedades analgésicas, bioestimulante celular e de modulação do processo inflamatório, qualificando esta irradiação eletromagnética laser como uma modalidade efetiva na busca da reparação tecidual (Assis et al., 2012a; ASSIS et al., 2012b).

Assim, o uso da PBMT por laser na biomodulação da cartilagem articular em processo degenerativo do tecido cartilaginoso, por meio de suas propriedades fotoquímicas e fotobiológicas, tem sido estudado por pesquisadores em todo o mundo e seus efeitos positivos na recuperação tecidual vêm sendo evidenciados (Xiang et al., 2020; Vassão et al., 2021).

\section{Metodologia}

Trata-se de uma revisão narrativa da literatura, de caráter qualitativo, descritivo exploratória, que é um tipo de estudo apropriado para descrever e discutir o desenvolvimento ou o "estado da arte" de um determinado assunto, sobre ponto de vista teórico ou contextual. Constituem, basicamente, de análise da literatura publicada de artigos de revistas impressas e/ou eletrônicas, na interpretação e análise crítica pessoal dos autores, permitindo assim, a aquisição e atualização do conhecimento sobre uma temática específica em curto espaço de tempo (Macedo et al., 2020).

Todos os estudos e relatórios primários que avaliaram os efeitos da PBMT na osteoartrite de joelho foram incluídos nesta revisão, independentemente de seus desenhos, assim como os principais estudos que descreveram os mecanismos de ação da terapia. Devido à novidade do projeto e aos poucos dados disponíveis sobre o assunto, não foram aplicados critérios de exclusão para evitar a falta de dados e maximizar a abrangência da pesquisa.

O processo de busca foi realizado de forma independente por dois revisores (LA. e Y.S). As bases de dados bibliográficas consultadas foram PubMed/MEDLINE, Biblioteca Virtual de Saúde (BVS), Web of Science e SciELO. A busca se deu por artigos baseados em evidências publicados até 10 de janeiro de 2021 e as principais palavras-chave utilizadas para a revisão foram: Fotobiomodulação; Fototerapia; Laser; Osteoartrite. Nenhuma limitação de idioma foi aplicada.

\section{Resultados e Discussão}

O presente estudo teve o intuito de mostrar as evidências disponíveis a respeito do mecanismos de ação e implicações clínicas da PBMT a laser no tratamento da OAJ. Os principais resultados desse trabalho mostram que a PBMT promove alterações metabólicas e estruturais e modula o processo inflamatório do tecido cartilaginoso em processo degenerativo como na OAJ.

\section{PBMT promove alterações metabólicas e estruturais da cartilagem na OAJ}

Pesquisas com modelos experimentais de OAJ evidenciaram que a PBMT promove uma série de modificações metabólicas e estruturais do tecido articular (Xiang et al., 2020; Vassão et al., 2021).

Sabe-se que a fisiopatologia da OA também está relacionada com a capacidade de resposta dos condrócitos frente ao estímulo lesivo. O início do processo de OA é caracterizado pelo aumento da taxa metabólica de condrócitos com subsequente proliferação dos mesmos com objetivo de reparar o tecido lesado (Pritzker et al., 2006; Goldring \& Goldring, 2006). Desta forma, a tentativa de reparo resulta em desorganização celular, hipercelularidade, condrócitos hipertrofiados, com subsequente morte por apoptose (Thomas et al., 2007).

Nos estudos avaliados foi possível observar que a PBMT a laser é capaz de exercer um efeito positivo no metabolismo do condrócito, impedindo a sua desorganização, morte por apoptose e consequentemente a evolução do processo degenerativo 
(Pritzker et al, 2006; Thomas et al, 2007). Lin et al. (2012), afirmaram que o laser terapêutico no comprimento de onda infravermelho $(810 \mathrm{~nm})$ preveniu a degradação do tecido articular e a apoptose de condrócitos, comprovado através da diminuição da expressão da caspase-3 da cartilagem de ratos submetidos à um modelo de indução de OAJ por transecção do ligamento cruzado anterior (TLCA). Ainda, Guo et al., 2011, demostraram que dez aplicações de laser (810 nm) foram efetivas em minimizar a expressão proteica de caspases (caspase-3 e 8), prevenindo a degradação da MEC do tecido cartilaginoso 6 semanas após modelo de OA por TLCA. Assim, acredita-se que a PBMT a laser possa ter regulado a expressão de proteínas apoptóticas e prevenindo a progressão da doença.

Ainda, é relatado que a PBMT a laser é eficaz em aumentar a síntese de componentes da MEC do tecido cartilaginoso como as proteoglicanas (Gottlieb et al., 2006) e mucopolissacarídeo (sulfato de condroitina) (Lin et al., 2006). Uma possível explicação para esses achados é que a irradiação com laser foi capaz de estimular os cromóforos dos condrócitos, que podem ter influenciado a fisiologia mitocondrial, afetando subsequentemente a síntese de várias proteínas que compõe a MEC do tecido articular (Karu et al., 2013). Estes resultados estão em concordância com Gottlieb et al. (2006), que também observou um aumento da quantidade de proteoglicanos na cartilagem articular de joelho de coelhos submetidos a TLCA após irradiação com laser $692,6 \mathrm{~nm}$, a $4 \mathrm{~J} / \mathrm{cm}^{2}$. Utilizando o mesmo modelo experimental, Oliveira et al. (2013) e dos Santos et al. (2014) identificaram que a PBMT a laser $(830 \mathrm{~nm})$ foi capaz de melhorar a organização de condrócitos, reduzir as alterações estruturais da superfície articular, minimizando assim o grau de lesão articular. Do mesmo modo, Bublitz et al. (2014) e Oliveira et al., (2013), demostraram que a PBMT a laser $(830 \mathrm{~nm}$ ) foi capaz de modular a progressão do processo degenerativo da OAJ, evidenciado por uma modulação dos condrócitos, aumento da síntese de proteoglicanas e uma melhor organização estrutural de cartilagem articular em relação ao grupo que não receberam tratamento.

Deste modo, a melhor organização estrutural e a menor dano articular observadas descritas nos trabalhos acima, levanos a inferir que a PBMT a laser favorece a homeostasia tecidual e modularam a atividade/função dos condrócitos, evitando assim progressão do processo degenerativo em curso.

\section{PBMT modula o processo inflamatório articular}

Além das diversas alterações estruturais e metabólicas evidenciadas no tecido articular durante a OAJ, pesquisas mostram que a degradação progressiva da articulação é mediada por citocinas inflamatórias, proteases e outros mediadores que intensificam o dano tecidual (Wojdasiewicz et al., 2014). Como descrito anteriormente, essas citocinas pró-inflamatórias, tais como TNF- $\alpha$ e IL-1 $\beta$, exercem efeitos catabólicos sobre o metabolismo dos condrócitos, deprimindo a síntese de proteoglicanos e colágeno tipo II e contribuem para o aumento da síntese e ativação de enzimas proteolíticas, tais como as MMP-13, responsáveis pela degradação dos componentes da matriz da cartilagem (Goldring \& Goldring; 2004). Ainda, essas citocinas estimulam a migração de células inflamatórias para o local da lesão, as quais tendem a produzir quantidades excessivas de outros mediadores inflamatórios (prostaglandina E2, óxido nítrico, radicais livres e outras citocinas inflamatórias) além de estimular a apoptose de condrócitos. De acordo com Cuellar et al., (2010), TNF- $\alpha$ e IL-1 $\beta$ possuem papéis centrais na manutenção do processo inflamatório e na destruição da cartilagem articular durante a OA.

Um grande número de estudos têm demonstrado que a PBMT a laser é capaz de reduzir o infiltrado inflamatório e enzimas responsáveis pela liberação de fatores quimiotáticos da inflamação, inibir a síntese de citocinas pró-inflamatórias e aumentar os níveis de enzimas antioxidantes em vários modelos de inflamação articular (Bublitz et al., 2014; Oliveira et al., 2013).

O estudo experimental de Soriano et al., (2006) evidenciaram redução dos níveis plasmáticos de TNF- $\alpha$ de ratos submetidos ao modelo experimental de OA por injeção intra-articular e posteriormente tratado com laser HeNe. Castano et al., (2007), demonstraram que a PBMT a laser $(810 \mathrm{~nm}$ ) reduziu o edema articular, os níveis de citocinas inflamatórias (IL-1 $\beta$ e 
TNF- $\alpha$ ) e marcadores inflamatórios plasmáticos (PGE2) em OAJ de ratos (induzida por zimosan). Da Rosa et al., (2012) avaliaram a ação do laser vermelho $(606 \mathrm{~nm})$ e infravermelho $(808 \mathrm{~nm})$ em modelo experimental de OA induzida por papaína. Os autores encontraram que ambos comprimentos de onda foram eficientes no processo de reparo da cartilagem lesada, entretanto a PBMT com laser infravermelho obteve melhores resultados na redução do exsudato inflamatório e tecido fibroso e favoreceu a formação de novos vasos sanguíneos.

O estudo de Alves et al., (2013) relataram que o laser infravermelho $(808 \mathrm{~nm})$ aplicado no joelho de ratos submetidos ao modelo de OA por papaína, foi eficaz em reduzir citocinas inflamatórias, tais como, IL-1 $\beta$, IL-6 e TNF- $\alpha$, correlacionando estes achados com a melhora histológica da cartilagem articular. Não obstante, Dos Santos et al., (2014), utilizando o mesmo modelo de indução de OA por papaína, observaram que uma única aplicação de laser (808 nm) foi eficiente em reduzir células inflamatórias e a expressão gênica de IL-1 $\beta$ e IL-6.

Ademais, atualmente, vários estudos têm evidenciado os efeitos positivos do PBMT a laser sobre as MMPs em modelo experimental de OA de joelho (Oliveira et al., 2013; Santos et al., 2013; Alves et al., 2014; Bublitz et al., 2014). Estes estudos demonstraram uma redução dessas enzimas proteolíticas de colágeno do tipo II após aplicação de irradiação laser.

Assim, fica evidente que a PBMT a laser é eficiente em equilibrar a síntese de algumas citocinas anti e próinflamatória e reduzir enzimas proteolíticas, inferindo assim uma modulação do processo inflamatório e severidade da OA.

\section{PBMT e suas implicações clínicas}

De maneira similar, estudos clínicos têm demonstrado que a PBMT tem capacidade de reduzir a dor, rigidez articular, edema articular, aumentando o desempenho funcional e melhora da qualidade de vida dos pacientes com OAJ (Rayeganil et al., 2012; Yurtkuran et al., 2007). Yamaura et al. (2009) demonstraram em seu estudo clínico de pacientes com OAJ, que a PBMT com comprimento de onda de $810 \mathrm{~nm}$, foi capaz de reduzir os níveis de TNF- $\alpha$, IL-1 $\beta$ e IL- 8 , favorecendo a redução da dor e dano articular. Alghabier et al., (2014) verificaram que 8 sessões de laser infravermelho $\left(850 \mathrm{~nm} ; 48 \mathrm{~J} / \mathrm{cm}^{2}, 6 \mathrm{~J}\right.$ por ponto) foi eficaz em reduzir a dor e aumentar a habilidade funcional de pacientes com OA de joelho (grau II e III).

Dessa forma, acredita-se que o aumento da viabilidades dos condrócitos associado à modulação do processo inflamatório após a irradiação são os responsáveis por promover analgesia e, consequentemente, proporcionar melhora da função articular (Soriano et al., 2006).

Ainda, foi possível identificar que a PBMT a laser é recomendada pela Sociedade Internacional de Pesquisa em Osteoartrite (OARSI) como opções de tratamento não farmacológico e não invasivo para OA (Zhang et al., 2010).

Sendo assim, a utilização da PBMT a laser como recursos terapêuticos para o tratamento da OA poderiam ser impostos. A adição desse recurso em doses e períodos adequados, os tornaria a reabilitação mais efetiva e viáveis principalmente por ser um método não invasivo e também apresenta custos relativamente baixos. Entretanto, existe a necessidade de estudos clínicos controlados e randomizados com a intenção de investigar e elucidar os melhores parâmetros de tratamento, visto que o potencial terapêutico desta modalidade é dependente da aplicação de parâmetros de tratamento adequados, principalmente comprimento de onda, densidade de energia, potência, irradiância, número e tempo da sessão (Assis et al., 2018; Assis et al., 2016).

\section{Conclusão}

Diante do exposto, nota-se que a PBMT a laser vêm surgindo como recursos promissores no tratamento da OAJ, pois reduzem a morte de condrócitos, estimulam a síntese de MEC e minimizam a síntese de mediadores pró-inflamatórios, atenuando o dano articular e consequentemente melhorando a dor, funcionalidade e qualidade de vida do indivíduo acometido. 
Assim, essa terapêutica pode constituir um excelente coadjuvante no tratamento da OAJ, uma vez que é um método não invasivo e também apresenta custos relativamente baixos.

Considerando os resultados promissores da fotobiomodulação na osteoartrite de joelho, destacamos a importância de novos ensaios experimentais e clínicos (controlados e randomizados) para o entendimento dos mecanismos fisiológicos, e principalmente a dosimetria ideal para uma aplicação segura em portadores de osteoartrite de joelho.

\section{Agradecimentos}

O presente trabalho foi realizado com apoio da Coordenação de Aperfeiçoamento de Pessoal de Nível Superior- Brasil (CAPES).

\section{Referências}

Afsar, E., Taspinar, F., Calik, B. B., Ozkan, Y., \& Gok, K. (2017). Use of the finite element analysis to determine stresses in the knee joints of osteoarthritis patients with different Q angles. Journal of the Brazilian Society of Mechanical Sciences and Engineering, 39(4), 1061-1067. https://doi.org/10.1007/s40430016-0636-1

Alves, A. C., Albertini, R., dos Santos, S. A., Leal-Junior, E. C., Santana, E., Serra, A. J., Silva, J. A., Jr, \& de Carvalho, P. (2014). Effect of low-level laser therapy on metalloproteinase MMP-2 and MMP-9 production and percentage of collagen types I and III in a papain cartilage injury model. Lasers in medical science, 29(3), 911-919. https://doi.org/10.1007/s10103-013-1427-X

Assis, L., Milares, L. P., Almeida, T., Tim, C., Magri, A., Fernandes, K. R., Medalha, C., \& Renno, A. C. (2016). Aerobic exercise training and low-level laser therapy modulate inflammatory response and degenerative process in an experimental model of knee osteoarthritis in rats. Osteoarthritis and cartilage, 24(1), 169-177. https://doi.org/10.1016/j.joca.2015.07.020.

Assis, L., Tim, C., Magri, A., Fernandes, K. R., Vassão, P. G., \& Renno, A. (2018). Interleukin-10 and collagen type II immunoexpression are modulated by photobiomodulation associated to aerobic and aquatic exercises in an experimental model of osteoarthritis. Lasers in medical science, 33(9), 1875-1882. https://doi.org/10.1007/s10103-018-2541-6

Benner, R. W., Shelbourne, K. D., Bauman, S. N., Norris, A., \& Gray, T. (2019). Knee Osteoarthritis: Alternative Range of Motion Treatment. The Orthopedic clinics of North America, 50(4), 425-432. https://doi.org/10.1016/j.ocl.2019.05.001

Bublitz, C., Medalha, C., Oliveira, P., Assis, L., Milares, L. P., Fernandes, K. R., ... \& Renno, A. C. M. (2014). Low-level laser therapy prevents degenerative morphological changes in an experimental model of anterior cruciate ligament transection in rats. Lasers in medical science, 29(5), 1669-1678. https:// doi $10.1007 / \mathrm{s} 10103-014-1546-\mathrm{Z}$

Castano, A. P., Dai, T., Yaroslavsky, I., Cohen, R., Apruzzese, W. A., Smotrich, M. H., \& Hamblin, M. R. (2007). Low-level laser therapy for zymosaninduced arthritis in rats: Importance of illumination time. Lasers in surgery and medicine, 39(6), 543-550. https://doi.org/10.1002/lsm.20516

Cuellar, V. G., Cuellar, J. M., Golish, S. R., Yeomans, D. C., \& Scuderi, G. J. (2010). Cytokine profiling in acute anterior cruciate ligament injury. Arthroscopy: the journal of arthroscopic \& related surgery: official publication of the Arthroscopy Association of North America and the International Arthroscopy Association, 26(10),1296-1301. https://doi.org/10.1016/j.arthro.2010.02.011

Da Rosa, A. S., dos Santos, A. F., da Silva, M. M., Facco, G. G., Perreira, D. M., Alves, A. C., Leal Junior, E. C., \& de Carvalho, P. (2012). Effects of lowlevel laser therapy at wavelengths of 660 and $808 \mathrm{~nm}$ in experimental model of osteoarthritis. Photochemistry and photobiology, 88(1), 161-166. https://doi.org/10.1111/j.1751-1097.2011.01032.x

Dos Santos, S. A., Alves, A. C. A., Leal-Junior, E. C. P., Albertini, R., de Paula Vieira, R., Ligeiro, A. P., ... \& de Carvalho, P. D. T. C. (2014). Comparative analysis of two low-level laser doses on the expression of inflammatory mediators and on neutrophils and macrophages in acute joint inflammation. Lasers in medical science, 29(3), 1051-1058. https:// doi 10.1007/s10103-013-1467-2

Ferreira, R. M., Duarte, J. A., \& Gonçalves, R. S. (2018). Non-pharmacological and non-surgical interventions to manage patients with knee osteoarthritis: An umbrella review. Non-pharmacological and non-surgical interventions to manage patients with knee osteoarthritis: An umbrella review. Acta reumatologica portuguesa, 43(3), 182-200.

French, H. P., Cusack, T., Brennan, A., White, B., Gilsenan, C., Fitzpatrick, M., O'Connell, P., Kane, D., Fitzgerald, O., \& McCarthy, G. M. (2009). Exercise and manual physiotherapy arthritis research trial (EMPART): a multicentre randomised controlled trial. BMC musculoskeletal disorders, 10, 9. https://doi.org/10.1186/1471-2474-10-9

Goldring, S. R., \& Goldring, M. B. (2004). The role of cytokines in cartilage matrix degeneration in osteoarthritis. Clinical orthopaedics and related research, (427 Suppl), S27-S36. https://doi.org/10.1097/01.blo.0000144854.66565.8f

Goldring, S. R., \& Goldring, M. B. (2006). Clinical aspects, pathology and pathophysiology of osteoarthritis. Journal of Musculoskeletal and Neuronal Interactions, 6(4), 376 .

Gottlieb, T., Jörgensen, B., Rohde, E., Müller, G., \& Scheller, E. E. (2006). The influence of irradiation with low-level diode laser on the proteoglycan content in arthrotically changed cartilage in rabbits. Medical laser application, 21(1), 53-59. https:// doi.org/10.1016/j.mla.2005.12.004 
Guo, H., Luo, Q., Zhang, J., Lin, H., Xia, L., \& He, C. (2011). Comparing different physical factors on serum TNF- $\alpha$ levels, chondrocyte apoptosis, caspase-3 and caspase-8 expression in osteoarthritis of the knee in rabbits. Joint Bone Spine, 78(6), 604-610. https://doi.org/10.1016/j.jbspin.2011.01.009

Hafez, A. R., Al-Johani, A. H., Zakaria, A. R., Al-Ahaideb, A., Buragadda, S., Melam, G. R., \& Kachanathu, S. J. (2013). Treatment of knee osteoarthritis in relation to hamstring and quadriceps strength. Journal of physical therapy science, 25(11), 1401-1405. https://doi.org/10.1589/jpts.25.1401.

Hamblin M. R. (2013). Can osteoarthritis be treated with light? Arthritis research \& therapy, 15(5), 120. https://doi.org/10.1186/ar4354

Hinman, R. S., Campbell, P. K., Lawford, B. J., Briggs, A. M., Gale, J., Bills, C., Kasza, J., Harris, A., French, S. D., Bunker, S. J., Forbes, A., \& Bennell, K. L. (2020). Does telephone-delivered exercise advice and support by physiotherapists improve pain and/or function in people with knee osteoarthritis? Telecare randomised controlled trial. British journal of sports medicine, 54(13), 790-797. https://doi.org/10.1136/bjsports-2019-101183

Hussain, S. M., Neilly, D. W., Baliga, S., Patil, S., \& Meek, R. (2016). Knee osteoarthritis: a review of management options. Scottish medical journal, 61(1), 7-16. https://doi.org/10.1177/0036933015619588

Jones, A., Silva, P. G., Silva, A. C., Colucci, M., Tuffanin, A., Jardim, J. R., \& Natour, J. (2012). Impact of cane use on pain, function, general health and energy expenditure during gait in patients with knee osteoarthritis: a randomised controlled trial. Annals of the rheumatic diseases, 71(2), 172-179. https://doi.org/10.1136/ard.2010.140178

Kapoor, M., Martel-Pelletier, J., Lajeunesse, D., Pelletier, J. P., \& Fahmi, H. (2011). Role of proinflammatory cytokines in the pathophysiology of osteoarthritis. Nature reviews. Rheumatology, 7(1), 33-42. https://doi.org/10.1038/nrrheum.2010.196

Karu T. Is it time to consider photobiomodulation as a drug equivalent?. Photomedicine and laser surgery, 31(5), 189-191. https://doi.org/10.1089/pho.2013.3510

Lespasio, M. J., Piuzzi, N. S., Husni, M. E., Muschler, G. F., Guarino, A., \& Mont, M. A. (2017). Knee Osteoarthritis: A Primer. The Permanente journal, 21, 16-183. https://doi.org/10.7812/TPP/16-183.

Lin, H. D., He, C. Q., Luo, Q. L., Zhang, J. L., \& Zeng, D. X. (2012). The effect of low-level laser to apoptosis of chondrocyte and caspases expression, including caspase-8 and caspase-3 in rabbit surgery-induced model of knee osteoarthritis. Rheumatology international, 32(3), 759-766. https://doi.org/10.1007/s00296-010-1629-5

Lin, Y. S., Huang, M. H., \& Chai, C. Y. (2006). Effects of helium-neon laser on the mucopolysaccharide induction in experimental osteoarthritic cartilage. Osteoarthritis and cartilage, 14(4), 377-383. https://doi.org/10.1016/j.joca.2005.10.010

Lu, T. W., Wei, I. P., Liu, Y. H., Hsu, W. C., Wang, T. M., Chang, C. F., \& Lin, J. G. (2010). Immediate effects of acupuncture on gait patterns in patients with knee osteoarthritis. Chinese medical journal, 123(2), 165-172. https://doi: 10.3760/cma.j.issn.0366-6999.2010.02.008

Macedo, D. B., Tim, C. R., Macedo, J. B., Macedo, G. B., Martignago, C. C. S., \& Garcia, L. A. (2020). Perspectiva terapéutica de la luz para el tratamiento del coronavirus. Research, Society and Development. http://dx.doi.org/10.33448/rsd-v9i8.6320

Oliveira, P., Santos, A. A., Rodrigues, T., Tim, C. R., Pinto, K. Z., Magri, A. M., Fernandes, K. R., Mattiello, S. M., Parizotto, N. A., Anibal, F. F., \& Rennó, A. C. (2013). Effects of phototherapy on cartilage structure and inflammatory markers in an experimental model of osteoarthritis. Journal of biomedical optics, 18(12), 128004. https://doi.org/10.1117/1.JBO.18.12.128004

Pelletier, J. P., Jovanovic, D. V., Lascau-Coman, V., Fernandes, J. C., Manning, P. T., Connor, J. R., ... \& Martel-Pelletier, J. (2000). Selective inhibition of inducible nitric oxide synthase reduces progression of experimental osteoarthritis in vivo: possible link with the reduction in chondrocyte apoptosis and caspase 3 level. Arthritis \& Rheumatism: Official Journal of the American College of Rheumatology, 43(6), 1290-1299. https:// doi.org/10.1002/15290131(200006)43:6<1290::AID-ANR11>3.0.CO;2-R

Perrot S. Osteoarthritis pain. Best practice \& research. Clinical rheumatology, 29(1), 90-97. https://doi.org/10.1016/j.berh.2015.04.017

Pritzker, K. P., Gay, S., Jimenez, S. A., Ostergaard, K., Pelletier, J. P., Revell, P. A., Salter, D., \& van den Berg, W. B. (2006). Osteoarthritis cartilage histopathology: grading and staging. Osteoarthritis and cartilage, 14(1), 13-29. https://doi.org/10.1016/j.joca.2005.07.014

Rayegani, S. M., Bahrami, M. H., Elyaspour, D., Saeedi, M., \& Sanjari, H. (2012). Therapeutic effects of low level laser therapy (LLLT) in knee osteoarthritis, compared to therapeutic ultrasound.

Renner, A. F., Carvalho, E., Soares, E., \& Mattiello-Rosa, S. (2006). The effect of a passive muscle stretching protocol on the articular cartilage. Osteoarthritis and cartilage, 14(2), 196-202. https://doi.org/10.1016/j.joca.2005.08.011

Shi, Q., Vaillancourt, F., Côté, V., Fahmi, H., Lavigne, P., Afif, H., Di Battista, J. A., Fernandes, J. C., \& Benderdour, M. (2006). Alterations of metabolic activity in human osteoarthritic osteoblasts by lipid peroxidation end product 4-hydroxynonenal. Arthritis research \& therapy, 8(6), R159. https://doi.org/10.1186/ar2066

Soriano, F., Campana, V., Moya, M., Gavotto, A., Simes, J., Soriano, M., \& Palma, J. (2006). Photobiomodulation of pain and inflammation in microcrystalline arthropathies: experimental and clinical results. Photomedicine and Laser Therapy, 24(2), 140-150. https:// doi.org/10.1089/pho.2006.24.140

Thomas, C. M., Fuller, C. J., Whittles, C. E., \& Sharif, M. (2007). Chondrocyte death by apoptosis is associated with cartilage matrix degradation. Osteoarthritis and cartilage, 15(1), 27-34. https://doi.org/10.1016/j.joca.2006.06.012

Trevisan, E. S., Martignago, C., Assis, L., Tarocco, J. C., Salman, S., Dos Santos, L., Liebano, R., \& Tim, C. R. (2020). Effectiveness of Led Photobiomodulation Therapy on Treatment With Knee Osteoarthritis: A Rat Study. American journal of physical medicine \& rehabilitation, 99(8), 725-732. https://doi.org/10.1097/PHM.0000000000001408

Tung-wu, L., Wei, I. P., Yen-hung, L., Wei-chun, H., Ting-ming, W., Chu-fen, C., \& Jaung-geng, L. (2010). Immediate effects of acupuncture on gait patterns in patients with knee osteoarthritis. Chinese Medical Journal, 123(2), 165-172. 
Research, Society and Development, v. 10, n. 1, e59410112072, 2021

(CC BY 4.0) | ISSN 2525-3409 | DOI: http://dx.doi.org/10.33448/rsd-v10i1.12072

Turkiewicz, A., Petersson, I. F., Björk, J., Hawker, G., Dahlberg, L. E., Lohmander, L. S., \& Englund, M. (2014). Current and future impact of osteoarthritis on health care: a population-based study with projections to year 2032. Osteoarthritis and cartilage, 22(11), 1826-1832. https://doi.org/10.1016/j.joca.2014.07.015

Vassão, P. G., Parisi, J., Penha, T., Balão, A. B., Renno, A., \& Avila, M. A. (2021). Association of photobiomodulation therapy (PBMT) and exercises programs in pain and functional capacity of patients with knee osteoarthritis (KOA): a systematic review of randomized trials. Lasers in medical science, 10.1007/s10103-020-03223-8. Advance online publication. https://doi.org/10.1007/s10103-020-03223-8

Vina, E. R., \& Kwoh, C. K. (2018). Epidemiology of osteoarthritis: literature update. Current opinion in rheumatology, 30(2), 160-167. https://doi.org/10.1097/BOR.0000000000000479

Wojdasiewicz, P., Poniatowski, Ł. A., \& Szukiewicz, D. (2014). The role of inflammatory and anti-inflammatory cytokines in the pathogenesis of osteoarthritis. Mediators of inflammation, 2014, 561459. https://doi.org/10.1155/2014/561459

Xiang, A., Deng, H., Cheng, K., Liu, H., Lin, L., Qu, X., Liu, S., \& Shen, X. (2020). Laser photobiomodulation for cartilage defect in animal models of knee osteoarthritis: a systematic review and meta-analysis. Lasers in medical science, 35(4), 789-796. https://doi.org/10.1007/s10103-019-02937-8

Yamaura, M., Yao, M., Yaroslavsky, I., Cohen, R., Smotrich, M., \& Kochevar, I. E. (2009). Low level light effects on inflammatory cytokine production by rheumatoid arthritis synoviocytes. Lasers in surgery and medicine, 41(4), 282-290. https://doi.org/10.1002/lsm.20766

Yurtkuran, M., Alp, A., Konur, S., Ozçakir, S., \& Bingol, U. (2007). Laser acupuncture in knee osteoarthritis: a double-blind, randomized controlled study. Photomedicine and laser surgery, 25(1), 14-20. https://doi.org/10.1089/pho.2006.1093

Zhang W, Moskowitz RW, Nuki G, et al. OARSI recommendations for the management of hip and knee osteoarthritis, Part II: OARSI evidence-based, expert consensus guidelines. Osteoarthritis Cartilage 2008; 16:137-62. Osteoarthritis and cartilage, 16(12), 1585-1589. https://doi.org/10.1016/j.joca.2008.04.019

Zhang, W., Nuki, G., Moskowitz, R. W., Abramson, S., Altman, R. D., Arden, N. K., \& Tugwell, P. (2010). OARSI recommendations for the management of hip and knee osteoarthritis: part III: Changes in evidence following systematic cumulative update of research published through January 2009. Osteoarthritis and cartilage, 18(4), 476-499. https://doi.org/10.1016/j.joca.2010.01.013 